\title{
Facial Pain
}

National Cancer Institute

\section{Source}

National Cancer Institute. Facial Pain. NCI Thesaurus. Code C79594.

Painful sensation in the face. 